\title{
MiXed SPECTRA FOR STABLE Signals FROM DISCRETE OBSERVATIONS
}

\author{
Rachid Sabre \\ Biogeosciences (UMR CNRS/uB 6282), \\ University of Burgundy, 26, Bd Docteur Petitjean, Dijon, France
}

\begin{abstract}
This paper concerns the continuous-time stable alpha symmetric processes which are inivitable in the modeling of certain signals with indefinitely increasing variance. Particularly the case where the spectral measurement is mixed: sum of a continuous measurement and a discrete measurement. Our goal is to estimate the spectral density of the continuous part by observing the signal in a discrete way. For that, we propose a method which consists in sampling the signal at periodic instants. We use Jackson's polynomial kernel to build a periodogram which we then smooth by two spectral windows taking into account the width of the interval where the spectral density is non-zero. Thus, we bypass the phenomenon of aliasing often encountered in the case of estimation from discrete observations of a continuous time process.
\end{abstract}

\section{KEYWORDS}

Spectral density, stable processes, periodogram, smoothing estimate, aliasing.

\section{INTRODUCTION}

The multiple applications where the random signals whose variance increases indefinitely impose the interest of using stable alpha processes by several research authors in various fields. In particular, Stable symmetric harmonizable processes and their properties have been widely studied by many authors such as [1] - [10] to name a few.

Concrete applications of stable symmetric processes cover a wide spectrum of fields such as: physics, biology, electronics and electricity, hydrology, economics, communications and radar applications., ...ect. See: [11]-[22]. In this paper we consider a symmetric alpha stable harmonizable process $X=\{X(t): t \in R\}$. Alternatively $X$ has the integral representation:

$$
X(t)=\int \exp [i(t \lambda)] d \xi(\lambda)
$$

where $1<\alpha<2$ and $\xi$ is a complex valued symmetric $\alpha$-stable random measure on $R$ with independent and isotropic increments. The control measure is defined by $m(A)=|\xi(A)|_{\alpha}^{\alpha}$ (see [4]) is called spectral measure. The estimation of the spectral density function was already studied in different cases: by E.Masry and S.Combanis [4] when the time of the process is continuous, by Sabre [23] when the time of the process is discrete and by R.Sabre [24]-[25]when the time of the process is p-adic.

In this paper we consider a general case where the spectral measure is the sum of an absolutely continuous measure with respect to Lebesgue measure and a discrete measure: 
Signal \& Image Processing: An International Journal (SIPIJ) Vol.12, No.5, October 2021

$$
d \mu(\lambda)=\phi(x) d x+\sum_{i=1}^{q} c_{i} \delta_{w_{i}}
$$

where $\delta$ is a Dirac measure, the specral density $\phi$ is nonnegative integrable and bounded function. $c_{i}$ is an unknown positive real number and $w_{i}$ is an unknown real number. Assume that $w_{i} \neq 0$. The discrete measurement comes from the repeated random energy jumps during the experimental measurements. Spectral density represents the distribution of the energy carried by the signal.

Our objective is to propose a nonparametric estimator of the spectral density after discrete sampling of the process $X(t)$. This work is motivated by the fact that in practice it is impossible to observe the process over a continuous time interval. However, we sampled the process at equidistant times, i.e., $t_{n}=n \tau, \tau>0$. It is known that aliasing of $\phi$ occurs. For more details about aliasing phenomenon, see [26]. To avoid this difficulty, we suppose that the spectral density $\phi$ is vanishing for $|\lambda|>\Omega$ where $\Omega$ is a nonnegative real number. We create an estimate of the spectral density based on smoothing methods. We show that it is asymptotically unbiased and consistent.

Briefly, the organization of this paper will be as follows: in the second section two technical lemmas will be presented as well as a preiodogram and we will show that this periodogram is an asymptotically unbiased but inconsistent estimator. In the third section, the periodogram will be smoothed by two well chosen spectral windows to estimate the spectral density at the jump points. We show that the smoothing periodogram is a consistent estimator. The fourth section gives conclusions and working perspectives.

\section{The Periodogram And its Proprieties}

In this section we give some basic notations and properties of the Jackson's polynomial kernel. Let $N$ is the size of sample of $X$. Let $k$ and $n$ are the numbers satisfying: $N-1=2 k(n-1) \quad$ with $\quad n \in N \quad k \in N \cup\left\{\frac{1}{2}\right\}$ if $k=\frac{1}{2} \quad$ then $\quad n=2 n_{1}-1, n_{1} \in N$.

The Jackson's polynomial kernel is defined by: $\left|H_{N}(\lambda)\right|^{\alpha}=\left|A_{N} H^{(N)}(\lambda)\right|^{\alpha}$ where

$$
H^{(N)}(\lambda)=\frac{1}{q_{k, n}}\left(\frac{\sin \frac{n \lambda}{2}}{\sin \frac{\lambda}{2}}\right)^{2 k} \text { with } q_{k, n}=\frac{1}{2 \pi} \int_{-\pi}^{\pi}\left(\frac{\sin \frac{n \lambda}{2}}{\sin \frac{\lambda}{2}}\right)^{2 k} d \lambda
$$

where $A_{N}=\left(B_{\alpha, N}\right)^{\frac{-1}{\alpha}}$ with $B_{\alpha, N}=\int_{-\pi}^{\pi}\left|H^{(N)}(\lambda)\right|^{\alpha} d \lambda$.

We cite two lemmas which are used in this paper. Their proof are given in [23].

Lemma 2.1 There is a non negative function $h_{k}$ such as: 
Signal \& Image Processing: An International Journal (SIPIJ) Vol.12, No.5, October 2021

$$
H^{(N)}(\lambda)=\sum_{m=-k(n-1)}^{k(n-1)} h_{k}\left(\frac{m}{n}\right) \cos (m \lambda)
$$

Lemma 2.2 Let $B_{\alpha, N}^{\prime}=\int_{-\pi}^{\pi}\left|\frac{\sin \frac{n \lambda}{2}}{\sin \frac{\lambda}{2}}\right|^{2 k \alpha} d \lambda \quad$ and $J_{N, \alpha}=\int_{-\pi}^{\pi}|u|^{\gamma}\left|H_{N}(u)\right|^{\alpha} d u$, where $\gamma \in] 0,2]$, then

and

$$
B_{\alpha, N}^{\prime}\left\{\begin{array}{l}
\geq 2 \pi\left(\frac{2}{\pi}\right)^{2 k \alpha} n^{2 k \alpha-1} \quad \text { if } \quad 0<\alpha<2 \\
\leq \frac{4 \pi k \alpha}{2 k \alpha-1} n^{2 k \alpha-1} \quad \text { if } \quad \frac{1}{2 k}<\alpha<2
\end{array}\right.
$$

$$
J_{N, \alpha} \leq\left\{\begin{array}{lll}
\frac{\pi^{\gamma+2 k \alpha}}{2^{2 k \alpha}(\gamma-2 k \alpha+1)} \frac{1}{n^{2 k \alpha-1}} & \text { if } & \frac{1}{2 k}<\alpha<\frac{\gamma+1}{2 k} \\
\frac{2 k \alpha \pi^{\gamma+2 k \alpha}}{2^{2 k \alpha}(\gamma+1)(2 k \alpha-\gamma-1)} \frac{1}{n^{\gamma}} & \text { if } & \frac{\gamma+1}{2 k}<\alpha<2
\end{array}\right.
$$

This paragraph gives a periodogram and develops its proprieties. Consider the process $X(t)$, defined in (1), observed at instants $t_{j}=j \tau, j=1,2, \ldots N$ and $\tau=\frac{2 \pi}{\omega}$, where $\omega$ is a real number strictly greater than $2 \Omega$. Define the periodogram $\hat{I}_{N}$ on $]-\Omega, \Omega[$ as follows:

$$
\hat{I}_{N}(\lambda)=C_{p, \alpha}\left|I_{N}(\lambda)\right|^{p}, \quad 0<p<\frac{\alpha}{2}
$$

Where

$$
I_{N}(\lambda)=[\tau]^{\frac{1}{\alpha}} A_{N} \operatorname{Re}\left[\sum_{n^{\prime}=-k(n-1)}^{n^{\prime}=k(n-1)} h_{k}\left(\frac{n^{\prime}}{n}\right) \exp \left\{-i\left(n^{\prime} \tau \lambda\right)\right\} X\left(n^{\prime} \tau+k(n-1) \tau\right)\right]
$$

The normalisation constant $C_{p, \alpha}$ is given by $C_{p, \alpha}=\frac{D_{p}}{F_{p, \alpha}\left[C_{\alpha}\right]^{p / \alpha}}$, with

$$
D_{p}=\int_{-\infty}^{\infty} \frac{1-\cos (u)}{|u|^{1+p}} d u \quad \text { and } \quad F_{p, \alpha}=\int_{-\infty}^{\infty} \frac{1-e^{-|u|^{a}}}{|u|^{1+p}} d u \text {. }
$$

\section{Lemma 2.3}


Signal \& Image Processing: An International Journal (SIPIJ) Vol.12, No.5, October 2021

The characteristic function of $I_{N}(\lambda), E \exp \left[\operatorname{irI}_{N}(\lambda)\right]$, converges to $\exp \left[-C_{\alpha}|r|^{\alpha}\left(\psi_{N, 1}(\lambda)+\psi_{N, 2}(\lambda)\right]\right.$. where

$$
\psi_{N, 1}(\lambda)=\int_{-\pi}^{\pi}\left|H_{N}(y-\tau \lambda)\right|^{\alpha} \phi\left(\frac{y}{\tau}\right) d y \text { and } \psi_{N, 2}(\lambda)=\sum_{i=1}^{q} c_{i}\left|H_{N}\left(w_{i}-\tau \lambda\right)\right|^{\alpha}
$$

\section{Proof}

From (1) and the expression of $I_{N}$, we have:

$$
I_{N}(\lambda)=[\tau]^{\frac{1}{\alpha}} A_{N} \operatorname{Re} \int_{R} \sum_{n^{\prime}=-k(n-1)}^{n^{\prime}=k(n-1)} h_{k}\left(\frac{n^{\prime}}{n}\right) \exp \left\{i\left[n^{\prime} \tau(\lambda-u)\right]\right\} \exp \{i[\tau u k(n-1)]\} d \xi(u) .
$$

Using [1] and the definition of the Jackson polynomial kernel we obtain that the characteristic function is the form:

$$
E \exp \left[\operatorname{irI}_{N}(\lambda)\right]=\exp \left[-C_{a}|r|^{a} \psi_{N}(\lambda)\right]
$$

where $\psi_{N}(\lambda)=\psi_{N, 1}(\lambda)+\psi_{N, 2}(\lambda)$ with

$$
\begin{array}{r}
\psi_{N, 1}(\lambda)=\int_{R}\left|H_{N}(v-\tau \lambda)\right|^{a} \phi\left(\frac{v}{\tau}\right) d v \text { and } \psi_{N, 2}(\lambda)=\sum_{i=1}^{q} c_{i}\left|H_{N}\left(w_{i}-\tau \lambda\right)\right|^{\alpha} \\
\psi_{N, 1}(\lambda)=\int_{R}\left|H_{N}(v-\tau \lambda)\right|^{a} \phi\left(\frac{v}{\tau}\right) d v=\sum_{j \in Z} \int_{(2 j-1) \pi}^{(2 j+1) \pi}\left|H_{N}(v-\tau \lambda)\right|^{a} \phi\left(\frac{v}{\tau}\right) d v .
\end{array}
$$

Putting $v=y-2 \pi j$ and using the fact that $H_{N}$ is $2 \pi$-periodic, we get $\psi_{N}(\lambda)=\sum_{j \in Z} \int_{-\pi}^{\pi}\left|H_{N}(y-\tau \lambda)\right|^{a} \phi_{j}(y) d y$, where $\phi_{j}(y)=\phi\left(\frac{y}{\tau}-\frac{2 \pi}{\tau} j\right)$. Let $j$ be an integer such that $-\Omega<\frac{y-2 \pi j}{\tau}<\Omega$. Since $\tau \Omega<\pi$ and $|y|<\pi$, we get $|j|<\frac{\tau \Omega}{2 \pi}+\frac{1}{2}<1$ and then $j=0$. Therefore

$$
\psi_{N, 1}(\lambda)=\int_{-\pi}^{\pi}\left|H_{N}(y-\tau \lambda)\right|^{\alpha} \phi\left(\frac{y}{\tau}\right) d y .
$$

Theorem 2.4 Let $-\Omega<\lambda<\Omega$ then $E\left[\hat{I}_{N}(\lambda)\right]=\left[\psi_{N}(\lambda)\right]^{\frac{p}{\alpha}}$,

\section{Proof}

From the following equality used in [4], for all real $x$ and $0<p<2$,

$$
|x|^{p}=D_{p}^{-1} \int_{-\infty}^{\infty} \frac{1-\cos (x u)}{|u|^{1+p}} d u=D_{p}^{-1} \operatorname{Re} \int_{-\infty}^{\infty} \frac{1-e^{i x u}}{|u|^{1+p}} d u,
$$


and replacing $x$ by $I_{N}$, we obtain

$$
\hat{I}_{N}(\lambda)=\frac{1}{F_{p, \alpha}\left[C_{a}\right]^{p / \alpha}} \operatorname{Re} \int_{-\infty}^{\infty} \frac{1-\exp \left\{i u I_{N}(\lambda)\right\}}{|u|^{1+p}} d u
$$

The equation (5) and the definition of the $F_{p, \alpha}$, give

$$
\begin{aligned}
E \hat{I}_{N}(\lambda) & =\frac{1}{F_{p, \alpha}\left[C_{a}\right]^{p / \alpha}} \int_{R} \frac{1-\exp \left\{-C_{a}|u|^{\alpha} \psi_{N}(\lambda)\right\}}{|u|^{1+p}} d u . \\
& =\left[\psi_{N}(\lambda)\right]^{p / \alpha} .
\end{aligned}
$$

\section{Smoothing Periodogram}

In order to obtain a consistent estimate of $[\phi(\lambda)]^{\frac{p}{\alpha}}$, we smooth the periodogram via spectral windows depending on whether $\tau \lambda$ is a jump point or $\operatorname{not}\left(\tau \lambda \neq w_{i}\right)$.

$$
f_{N}(\lambda)=\left\{\begin{array}{cc}
f_{N}^{(1)}(\lambda) \text { if } \tau \lambda \notin\left\{w_{1}, w_{2}, \cdots, w_{q}\right\} \\
\frac{f_{N}^{(2)}(\lambda)-c f_{N}^{(1)}(\lambda)}{1-c} \quad \text { else }
\end{array}\right.
$$

where $f_{N}^{(1)}(\lambda)=\int_{-\pi}^{\pi} W_{N}^{(1)}(\lambda-u) \hat{I}_{N}(u) d u$ and $f_{N}^{(2)}(\lambda)=\int_{-\pi}^{\pi} W_{N}^{(2)}(\lambda-u) \hat{I}_{N}(u) d u$.

The spectral windows $W_{N}^{(1)}$ and $W_{N}^{(2)}$ are defined by: $W_{N}^{(1)}(x)=M_{N}^{(1)} W\left(M_{N}^{(1)} x\right)$ and $W_{N}^{(2)}(x)=M_{N}^{(2))} W\left(M_{N}^{(2)} x\right)$ with $W$ is an even nonnegative, continuous function, vanishing for $|\lambda|>1$ such that $\int_{-1}^{1} W(u) d u=1$. The bandwidths $\left.M_{N}^{(1)}\right)$ and $\left.M_{N}^{(2)}\right)$ satisfying: $c=\frac{M_{N}^{(2)}}{M_{N}^{(1)}}$ $\lim _{N \rightarrow \infty} M_{N}^{(i)}=+\infty, \quad \lim _{N \rightarrow \infty} \frac{M_{N}^{(i)}}{N}=0 \quad$ for $i=1,2, \quad \lim _{N \rightarrow+\infty} \frac{M_{N}^{(2)}}{M_{N}^{(1)}}=0$ and such that $\left.W\left(M_{N}^{(2)} \theta\right)=W\left(M_{N}^{(1)} \theta\right) \forall \theta \in\right]-\frac{1}{M_{N}^{(1)}}, \frac{1}{M_{N}^{(1)}}[$.

Th following theorem shows that $f_{N}(\lambda)$ is an asymptotically unbiased estimator of $[\phi(\lambda)]^{\frac{p}{\alpha}}$ for $-\Omega<\lambda<\Omega$ and $\tau \lambda \notin\left\{w_{1}, w_{2}, \cdots, w_{q}\right\}$.

\section{Theorem 3.1}

Let $-\Omega<\lambda<\Omega$, such that $\tau \lambda \notin\left\{w_{1}, w_{2}, \cdots, w_{q}\right\}$. Then, $E\left[f_{N}(\lambda)\right]-[\phi(\lambda)]^{\frac{p}{\alpha}}=\mathrm{o}(1)$. 
Signal \& Image Processing: An International Journal (SIPIJ) Vol.12, No.5, October 2021

If $\phi$ satisfies the hypothesis $|\phi(x)-\phi(y)| \leq c s t e|(x-y)|^{-\gamma}$, with $\gamma<2 k \alpha-1$, then,

$$
E\left[f_{N}(\lambda)\right]-[\phi(\lambda)]^{\frac{p}{\alpha}}=\left\{\begin{array}{c}
O\left(\frac{1}{n^{(2 k \alpha-1)}}+\frac{1}{M_{N}^{(1) \gamma}}\right) \quad \text { if } \quad \lambda \neq 0 \\
O\left(\frac{1}{M_{N}^{(1)} n^{(2 k \alpha-1)}}+\frac{1}{M_{N}^{(1) \gamma}}+\frac{1}{n^{2 k \alpha-1}}\right) \quad \text { if } \quad \lambda=0
\end{array} .\right.
$$

\section{Proof}

It is easy to see that:

$$
E\left[f_{N}(\lambda)\right]=\int_{R} M_{N}^{(1)} W\left[M_{N}^{(1)}(\lambda-u)\right] E\left[\hat{I}_{N}(u)\right] d u
$$

Let $\quad M_{N}^{(1)}(\lambda-u)=v$, we obtain:

$$
E\left[f_{N}(\lambda)\right]=\int_{-1}^{1} W(v)\left[\psi_{N}\left(\lambda-\frac{v}{M_{N}^{(1)}}\right)\right]^{\frac{p}{\alpha}} d v
$$

Since $\int_{-1}^{1} W(u) d u=1$ and the inequality (3), we get:

$$
\left|E\left[f_{N}(\lambda)\right]-[\phi(\lambda)]^{\frac{p}{\alpha}}\right| \leq \int_{-1}^{1} W(v)\left|\psi_{N}\left(\lambda-\frac{v}{M_{N}^{(1)}}\right)-\phi(\lambda)\right|^{\frac{p}{\alpha}} d v .
$$

As $\frac{p}{\alpha}<1$, we obtain

$$
\left|\psi_{N}\left(\lambda-\frac{v}{M_{N}^{(1)}}\right)-\phi(\lambda)\right|^{\frac{p}{\alpha}} \leq\left|\psi_{N, 1}\left(\lambda-\frac{v}{M_{N}^{(1)}}\right)-\phi(\lambda)\right|^{\frac{p}{\alpha}}+\left|\psi_{N, 2}\left(\lambda-\frac{v}{M_{N}^{(1)}}\right)\right|^{\frac{p}{\alpha}}
$$

We now examine the limit of $\psi_{N, 1}\left(\lambda-\frac{v}{M_{N}^{(1)}}\right)$. From (3) we get:

$$
\psi_{N, 1}\left(\lambda-\frac{v}{M_{N}^{(1)}}\right)=\int_{-\infty}^{\infty}\left|H_{N}\left(u-\tau\left(\lambda-\frac{v}{M_{N}^{(1)}}\right)\right)\right|^{\alpha} \phi\left(\frac{u}{\tau}\right) d u
$$

Let $u-\tau\left(\lambda-\frac{v}{M_{N}^{(1)}}\right)=y$, we obtain: 
Signal \& Image Processing: An International Journal (SIPIJ) Vol.12, No.5, October 2021

$$
\begin{aligned}
\psi_{N, 1}\left(\lambda-\frac{v}{M_{N}^{(1)}}\right) & =\int_{R}\left|H_{N}(y)\right|^{\alpha} \phi\left(\lambda-\frac{v}{M_{N}^{(1)}}+\frac{y}{\tau}\right) d y \\
& =\sum_{j \in Z} \int_{(2 j-1) \pi}^{(2 j+1) \pi}\left|H_{N}(y)\right|^{\alpha} \phi\left(\lambda-\frac{v}{M_{N}^{(1)}}+\frac{y}{\tau}\right) d y .
\end{aligned}
$$

Let $y-2 j \pi=s$. Since $\left|H_{N}(.)\right|^{\alpha}$ is $2 \pi$-periodic function, we get

$$
\psi_{N, 1}\left(\lambda-\frac{v}{M_{N}^{(1)}}\right)=\sum_{j \in Z} \int_{-\pi}^{+\pi}\left|H_{N}(s)\right|^{\alpha} \phi\left(\lambda-\frac{v}{M_{N}^{(1)}}+\frac{s}{\tau}+\frac{2 \pi}{\tau} j\right) d s
$$

The function $\phi$ is uniformly continuous on $[-\Omega, \Omega]$ and since $\left|H_{N}\right|^{\alpha}$ is a kernel, the right hand side of the last equality converges to $\sum_{j \in Z} \phi\left(\lambda+\frac{2 \pi j}{\tau}\right)$. Let $j$ be an integer such that $-\Omega<\frac{\tau \lambda+2 \pi j}{\tau}<\Omega$. The definition of $\tau$ implies that $|\tau \lambda|<|\tau \Omega|<\pi$. It is easy to see that $|j|<1$ and then $j=0$. Since $H_{N}^{(1)}$ is a kernel, we obtain that $\psi_{N, 1}\left(\lambda-\frac{v}{M_{N}^{(1)}}\right)$ converges to $\phi(\lambda)$. On the other hand, $\quad \psi_{N, 2}\left(\lambda-\frac{v}{M_{N}^{(1)}}\right)=\sum_{i=1}^{q}\left|H_{N}^{(1)}\left(w_{i}-\tau\left(\lambda-\frac{v}{M_{N}^{(1)}}\right)\right)\right|^{\alpha}$

Since $w_{i}$ is different from $\tau \lambda$ and from the lemma 2.2, we get $\psi_{N, 2}\left(\lambda-\frac{v}{M_{N}^{(1)}}\right) \leq\left[2 \pi\left(\frac{2}{\pi}\right)^{2 k \alpha} n^{2 k \alpha-1}\right]^{-1} \frac{1}{c t e} \sum_{i=1}^{q} c_{i}$, where cte $=\inf \left|\sin \left(\frac{w_{i}-\tau\left(\lambda-\frac{v}{M_{N}^{(1)}}\right)}{2}\right)\right|$

Therefore, $\psi_{N, 2}\left(\lambda-\frac{v}{M_{N}^{(1)}}\right)=O\left(\frac{1}{n^{2 k \alpha-1}}\right)$. Thus, we have $E\left[f_{N}(\lambda)\right]-[\phi(\lambda)]^{\frac{p}{\alpha}}=o(1)$.

The rate of convergence:

Assume that the spectral density $\phi$ satisfies the hypothesis $H$. Let $F=\left|\operatorname{Bias}\left(f_{N}(\lambda)\right)\right|=\left|E\left[f_{N}(\lambda)\right]-[\phi(\lambda)]^{p / \alpha}\right|$. It follows that

$$
F \leq \frac{p}{2 \alpha} \int_{-1}^{1} W(v)\left[\left[\psi_{N}\left(\lambda-\frac{v}{M_{N}^{(1)}}\right)\right]^{\frac{p}{\alpha}-1}+[\phi(\lambda)]^{\frac{p}{\alpha}-1}\right]\left|\psi_{N}\left(\lambda-\frac{v}{M_{N}^{(1)}}\right)-\phi(\lambda)\right| d v
$$


Signal \& Image Processing: An International Journal (SIPIJ) Vol.12, No.5, October 2021

Since $\psi_{N}\left(\lambda-\frac{v}{M_{N}}\right)$ converges to $\phi(\lambda)$, in order to study the rate of the convergence for $F$ we examine the rate of convergence of $\int_{-1}^{1} W(v)\left|\psi_{N}\left(\lambda-\frac{v}{M_{N}^{(1)}}\right)-\phi(\lambda)\right| d v$. Indeed, from (3), we obtain $\psi_{N}\left(\lambda-\frac{v}{M_{N}^{(1)}}\right)=\int_{-\pi}^{\pi}\left|H_{N}\left(y-\tau \lambda+\frac{\tau v}{M_{N}^{(1)}}\right)\right|^{\alpha} \phi\left(\frac{y}{\tau}\right) d y$.

Let $\Delta\left(\psi_{N}, \phi\right)=\psi_{N}\left(\lambda-\frac{v}{M_{N}^{(1)}}\right)-\phi(\lambda)$. Putting $t=-\left(y-\tau \lambda+\frac{\tau v}{M_{N}^{(1)}}\right)$ and using the condition $H$, we get $\left|\Delta\left(\psi_{N}, \phi\right)\right| \leq C_{1} \int_{\tau \lambda-\frac{\tau \nu}{M_{N}^{(1)}}-\pi}^{\frac{\tau \lambda-\frac{\tau v}{M_{N}^{(1)}}+\pi}{M_{N}}}\left|H_{N}(t)\right|^{\alpha}\left|\frac{v}{M_{N}^{(1)}}+\frac{t}{\tau}\right|^{\gamma} d t$.

We can maximize as follows:

$$
\begin{aligned}
\int_{-1}^{1} W(v)\left|\Delta\left(\psi_{N}, \phi\right)\right| d v & \leq 2^{\gamma} C_{1}\left|\frac{1}{M_{N}^{(1)}}\right|^{\gamma} \int_{-1}^{1} W(v)|v|^{\gamma} d v \\
& +2^{\gamma} \frac{C_{1}}{\tau^{\gamma}} \int_{-1}^{1} W(v) \int_{\tau_{1} \lambda-\frac{\tau_{N}}{M_{N}^{(1)}}-\pi}^{\tau \lambda-\frac{\tau_{1} v}{M_{N}^{(1)}+\pi}}\left|H_{N}(t)\right|^{\alpha}|t|^{\gamma} d t d v
\end{aligned}
$$

The second integral of the right hand side is bounded as follows:

$$
\begin{aligned}
\int_{\tau \lambda-\frac{\tau v}{M_{N}^{(1)}}-\pi}^{\tau \lambda-\frac{\tau v}{M_{N}^{(1)}+\pi}}\left|H_{N}(t)\right|^{\alpha}|t|^{\gamma} d t & \leq \int_{-|\tau \lambda|-\left|\frac{\tau v}{M_{N}^{(1)}}\right|-\pi}^{-\pi}\left|H_{N}(t)\right|^{\alpha}|t|^{\gamma} d t \\
& +\int_{-\pi}^{\pi}\left|H_{N}(t)\right|^{\alpha}|t|^{\gamma} d t \\
& +\int_{\pi}^{|\tau \lambda|+\mid \frac{\tau v}{M_{N}^{(1)}}++\pi}\left|H_{N}(t)\right|^{\alpha}|t|^{\gamma} d t .
\end{aligned}
$$

Since $\left|H_{N}().\right|$ is even, the first and the last integrals in the right hand side of (9) are equal. As $\frac{\tau \nu}{M_{N}}$ converges to zero and $\tau \lambda<\tau \Omega<\pi$, for a large $N$ we have: 
Signal \& Image Processing: An International Journal (SIPIJ) Vol.12, No.5, October 2021

$$
\begin{aligned}
\int_{\pi}^{|\tau \lambda|+\left|\frac{\tau v}{M_{N}^{(1)}}\right|+\pi}\left|H_{N}(t)\right|^{\alpha}|t|^{\gamma} d t & \leq(2 \pi)^{\gamma} \int_{\pi}^{|\tau \lambda|+\left|\frac{\tau v}{M_{N}^{(1)}}\right|+\pi}\left|H_{N}(t)\right|^{\alpha} d t \\
& \leq \frac{(2 \pi)^{\gamma}}{B_{\alpha, N}^{\prime}} \frac{|\tau \lambda|+\frac{\tau}{M_{N}^{(1)}}}{\left|\sin \left(\pi+|\tau \lambda|+\frac{\tau}{M_{N}^{(1)}}\right)\right|^{2 k \alpha}}
\end{aligned}
$$

The lemma 2.1 gives:

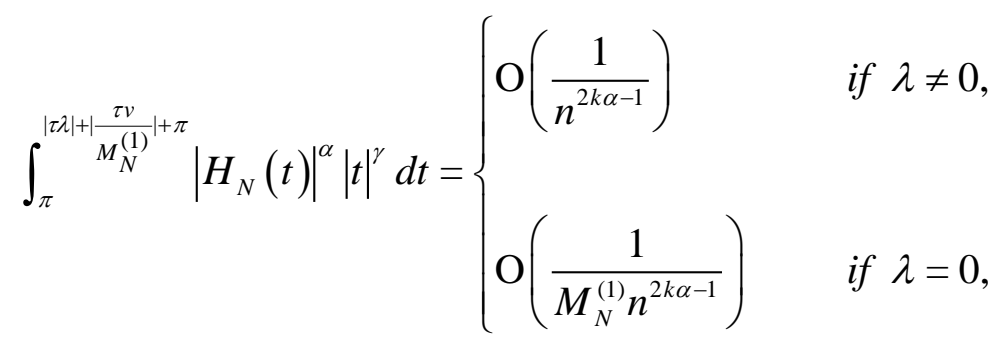

Thus, we obtain the result of the theorem.

Theorem 3.2. Let $\lambda$ a real nuber belonging to $]-\Omega, \Omega\left[\right.$, and $\tau \lambda=w_{i}$. Choose $k$ such that $\lim _{N \rightarrow \infty} \frac{\left(M_{N}^{(1)}\right)^{2 k \alpha}}{n^{2 k \alpha-1}}=0$. Then,

i) $\quad E\left[f_{N}(\lambda)\right]-[\phi(\lambda)]^{\frac{p}{\alpha}}=o(1)$

ii) If $\phi$ satisfiies the hypothesis $|\varphi(x)-\varphi(y)| \leq c s t e|(x-y)|^{-\gamma}$, with $\frac{\gamma+1}{2 k}<\alpha<2$, then

$$
\left|E\left[f_{N}(\lambda)\right]-[\phi(\lambda)]^{\frac{p}{\alpha}}\right|= \begin{cases}O\left(\frac{1}{\left(M_{N}^{(2)}\right)^{\gamma}}+\frac{\left(M_{N}^{(1)}\right)^{2 k \alpha}}{n^{2 k \alpha-1}}\right) \quad \text { if } & 0<\gamma \leq 1 \\ O\left(\frac{1}{M_{N}^{(2)}}+\frac{\left(M_{N}^{(1)}\right)^{2 k \alpha}}{n^{2 k \alpha-1}}\right) \text { if } & 1<\gamma \leq 2\end{cases}
$$

\section{Proof :}

The form of estimator gives: 
Signal \& Image Processing: An International Journal (SIPIJ) Vol.12, No.5, October 2021

$E\left[f_{N}(\lambda)\right]=\int_{-\pi}^{\pi} \frac{W_{N}^{(2)}(\lambda-u)-\frac{M_{N}^{(2)}}{M_{N}^{(1)}} W_{N}^{(1)}(\lambda-u)}{1-\frac{M_{N}^{(2)}}{M_{N}^{(1)}}}\left[\psi_{N}(u)\right]^{\frac{p}{\alpha}} d u$

$E\left[f_{N}(\lambda)\right]=\int_{-\pi}^{\lambda-\frac{1}{M_{N}^{(1)}}}+\int_{\lambda-\frac{1}{M_{N}^{(1)}}}^{\lambda+\frac{1}{M_{N}^{(1)}}}+\int_{\lambda+\frac{1}{M_{N}^{(1)}}}^{\pi}=E_{1}+E_{2}+E_{3}$

Put $\lambda-u=v, \quad E_{2}=\int_{-\frac{1}{M_{N}^{(1)}}}^{\frac{1}{M_{N}^{(1)}}} \frac{M_{N}^{(2)}\left[W\left[M_{n}^{(2)} v\right]-W\left[M_{N}^{(1)} v\right]\right]}{1-\frac{M_{N}^{(2)}}{M_{N}^{(1)}}}\left[\psi_{N}(\lambda-v)\right]^{\frac{p}{\alpha}} d v$. Therefore $E_{2}=0$

for a large $\mathrm{N}$.

$$
\begin{aligned}
& E_{1}=\frac{M_{N}^{(2)}}{1-\frac{M_{N}^{(2)}}{M_{N}^{(1)}}} \int_{-\pi}^{\lambda-\frac{1}{M_{N}^{(1)}}} W\left[M_{N}^{(2)}(\lambda-u)\right]\left[\psi_{N}(u)\right]^{\frac{p}{\alpha}} d u- \\
& \frac{M_{N}^{(2)}}{1-\frac{M_{N}^{(2)}}{M_{N}^{(1)}}} \int_{-\pi}^{\lambda-\frac{1}{M_{N}^{(1)}}} W\left[M_{N}^{(1)}(\lambda-u)\right]\left[\psi_{N}(u)\right]^{\frac{p}{\alpha}} d u
\end{aligned}
$$

Put $M_{N}^{(2)}(\lambda-u)=v$ in the first integral and put $M_{N}^{(1)}(\lambda-u)=w$, in the second integral and for a large $N$, we have $M_{N}^{(1)}(\lambda+\pi)>1$ et $M_{N}^{(2)}(\lambda+\pi)>1$. As $W$ is null outside of [-1,1], for large $N$, the second integral of $E_{1}$ is zero.Therefore,

$$
\begin{gathered}
E_{1}=\frac{1}{1-\frac{M_{N}^{(2)}}{M_{N}^{(1)}}} \int_{\frac{M_{N}^{(2)}}{M_{N}^{(1)}}}^{1} W(v)\left[\psi_{N}\left(\lambda-\frac{v}{M_{N}^{(2)}}\right)\right]^{\frac{p}{\alpha}} d v . \\
E_{3}=\frac{M_{N}^{(2)}}{1-\frac{M_{N}^{(2)}}{M_{N}^{(1)}}} \int_{\lambda+\frac{1}{M_{N}^{(1)}}}^{\pi} W\left[M_{N}^{(2)}(\lambda-u)\right]\left[\psi_{N}(u)\right]^{\frac{p}{\alpha}} d u-\frac{M_{N}^{(2)}}{1-\frac{M_{N}^{(2)}}{M_{N}^{(1)}}} \int_{\lambda+\frac{1}{M_{N}^{(1)}}}^{\pi} W\left[M_{N}^{(1)}(\lambda-u)\right]\left[\psi_{N}(u)\right]^{\frac{p}{\alpha}} d u
\end{gathered}
$$

Putting $M_{N}^{(2)}(\lambda-u)=v$ in the first integral and $M_{N}^{(1)}(\lambda-u)=w$ in the second integral, we obtain

$$
E_{3}=\frac{1}{1-\frac{M_{N}^{(2)}}{M_{N}^{(1)}}} \int_{M_{N}^{(2)}(\lambda-\pi)}^{-\frac{M_{N}^{(2)}}{M^{(1)}}} W(v)\left[\psi_{N}\left(\lambda-\frac{v}{M_{N}^{(2)}}\right)\right]^{\frac{p}{\alpha}} d v-\frac{\frac{M_{N}^{(2)}}{M_{N}^{(1)}}}{1-\frac{M_{N}^{(2)}}{M_{N}^{(1)}}} \int_{M_{N}^{(1)}(\lambda-\pi)}^{-1} W(w)\left[\psi_{N}\left(\lambda-\frac{w}{M_{N}^{(1)}}\right)\right]^{\frac{p}{\alpha}} d w .
$$


Signal \& Image Processing: An International Journal (SIPIJ) Vol.12, No.5, October 2021

For large $N$ we have, $M_{N}^{(2)}(\lambda-\pi)<-1$ and $M_{N}^{(1)}(\lambda-\pi)<-1$.

$$
E_{3}=\frac{1}{1-\frac{M_{N}^{(2)}}{M_{N}^{(1)}}} \int_{\frac{M_{N}^{(2)}}{M_{N}^{(1)}}}^{1} W(v)\left[\psi_{N}\left(\lambda+\frac{v}{M_{N}^{(2)}}\right)\right]^{\frac{p}{\alpha}} d v
$$

It is easy to show that for a large $N$

$$
\begin{gathered}
\frac{1}{1-\frac{M_{N}^{(2)}}{M_{N}^{(1)}}} \int_{\frac{M_{N}^{(2)}}{M_{N}^{(1)}}}^{1} W(v) d v=\frac{1}{2} \\
\left|E\left[f_{N}(\lambda)\right]-[\phi(\lambda)]^{\frac{p}{\alpha}}\right|=\left|E_{1}+E_{3}-[\phi(\lambda)]^{\frac{p}{\alpha}}\right| \leq\left|E_{1}-\frac{1}{2}[\phi(\lambda)]^{\frac{p}{\alpha}}\right|+\left|E_{3}-\frac{1}{2}[\phi(\lambda)]^{\frac{p}{\alpha}}\right|
\end{gathered}
$$

From (10) and (12), for a large $N$, we have

$$
\left|E_{1}-\frac{1}{2}[\phi(\lambda)]^{\frac{p}{\alpha}}\right| \leq \frac{1}{1-\frac{M_{N}^{(2)}}{M_{N}^{(1)}}} \int_{\frac{M_{N}^{(2)}}{M_{N}^{(1)}}}^{1} W(v)\left|\psi_{N, 1}\left(\lambda-\frac{v}{M_{N}^{(2)}}\right)+\psi_{N_{2}}\left(\lambda-\frac{v}{M_{N}^{(2)}}\right)-\phi(\lambda)\right|^{\frac{p}{\alpha}} d v
$$

As $\frac{p}{\alpha}<1$, we obtain

$$
\begin{aligned}
\left|E_{1}-\frac{1}{2}[\phi(\lambda)]^{\frac{p}{\alpha}}\right| \leq & \frac{1}{1-\frac{M_{N}^{(2)}}{M_{N}^{(1)}}} \int_{\frac{M_{N}^{(2)}}{M_{N}^{(1)}}}^{1} W(v)\left|\psi_{N, 1}\left(\lambda-\frac{v}{M_{N}^{(2)}}\right)-\phi(\lambda)\right|^{\frac{p}{\alpha}} d v \\
& +\frac{1}{1-\frac{M_{N}^{(2)}}{M_{N}^{(1)}}} \int_{\frac{M_{N}^{(2)}}{M_{N}^{(1)}}}^{1} W(v)\left|\psi_{N, 2}\left(\lambda-\frac{v}{M_{N}^{(2)}}\right)\right|^{\frac{p}{\alpha}} d v .
\end{aligned}
$$

On the other hand,

$$
\int_{\frac{M_{N}^{(2)}}{M_{N}^{(1)}}}^{1} W(v)\left|\psi_{N, 1}\left(\lambda \pm \frac{v}{M_{N}^{(2)}}\right)-\phi(\lambda)\right|^{\frac{p}{\alpha}} d v \leq \int_{0}^{1} W(v)\left|\psi_{N, 1}\left(\lambda \pm \frac{v}{M_{N}^{(2)}}\right)-\phi(\lambda)\right|^{\frac{p}{\alpha}} d v
$$

For all $\lambda$ belonging to $]-\pi, \pi\left[\psi_{N, 1}\left(\lambda \pm \frac{v}{M_{N}^{(2)}}\right)\right.$ converges to $\phi(\lambda)$, uniformly in $v \in[-1,1]$. Therefore, $\frac{1}{1-\frac{M_{N}^{(2)}}{M_{N}^{(1)}}} \int_{\frac{M_{N}^{(2)}}{M_{N}^{(1)}}}^{1} W(v)\left|\psi_{N, 1}\left(\lambda \pm \frac{v}{M_{N}^{(2)}}\right)-\phi(\lambda)\right|^{\frac{p}{\alpha}} d v$ converge to zero. 
Signal \& Image Processing: An International Journal (SIPIJ) Vol.12, No.5, October 2021

Since $\tau \lambda=w_{i}, \quad \psi_{N, 2}\left(\lambda_{i} \pm \frac{v}{M_{N}^{(2)}}\right) \leq \sum_{m=1}^{q} \frac{a_{m}}{B_{\alpha, N}^{\prime}} \frac{1}{\left|\sin \left[\frac{1}{2}\left(w_{i} \pm \frac{\tau v}{M_{N}^{(2)}}-w_{m}\right)\right]\right|^{2 k \alpha}}$

$$
+\frac{a_{i}}{B_{\alpha, N}^{\prime}} \frac{1}{\left.\sin \left[\frac{1}{2}\left( \pm \frac{\tau \nu}{M_{N}^{(2)}}\right)\right]\right|^{2 k \alpha}}
$$

$$
\text { For all } m \neq i, \lim _{N \rightarrow+\infty} \sup _{v \in[-1,1]} \frac{1}{\left|\sin \left[\frac{1}{2}\left(w_{i} \pm \frac{\tau v}{M_{N}^{(2)}}-w_{m}\right)\right]\right|^{2 k \alpha}}=\frac{1}{\left|\sin \left[\frac{1}{2}\left(w_{i}-w_{m}\right)\right]\right|^{2 k \alpha}} \text {. }
$$

Thus, for large N, we get

$\sum_{m=1}^{q} \frac{a_{m}}{B_{\alpha, N}^{\prime}} \frac{1}{\left|\sin \left[\frac{1}{2}\left(w_{i} \pm \frac{\tau v}{M_{N}^{(2)}}-w_{m}\right)\right]\right|^{2 k \alpha}} \leq\left(\varepsilon+\frac{1}{\inf _{m \in\{1,2, \cdots, q\}-\{i\}}\left|\sin \left[\frac{1}{2}\left(w_{i}-w_{m}\right)\right]\right|^{2 k \alpha}} \sum_{m=1}^{q} \frac{a_{m}}{B_{\alpha, N}^{\prime}}\right.$

The lemma 2.1 gives $\sum_{m=1}^{q} \frac{a_{m}}{B_{\alpha, N}^{\prime}} \frac{1}{\left.\sin \left[\frac{1}{2}\left(w_{i} \pm \frac{\tau v}{M_{N}^{(2)}}-w_{m}\right)\right]\right|^{2 k \alpha}}=O\left(\frac{1}{n^{2 k \alpha-1}}\right)$.

For large $N$, we have $\sup _{v \in] 0,1]}\left|\frac{\tau v}{M_{N}^{(2)}}\right|=\frac{\tau}{M_{N}^{(2)}}<\pi$. Consequently

$$
\frac{a_{i}}{B_{\alpha, N}^{\prime}}\left|\sin \left[\frac{1}{2}\left( \pm \frac{\tau v}{M_{N}^{(2)}}\right)\right]\right|^{2 k \alpha} \leq \frac{a_{i}}{B_{\alpha, N}^{\prime}} \frac{\pi^{2 k \alpha}}{\left|\frac{\tau v}{M_{N}^{(2)}}\right|^{2 k \alpha}}
$$

As $\quad \frac{\tau}{M_{N}^{(1)}} \leq \frac{\tau v}{M_{N}^{(2)}} \leq \frac{\tau}{M_{N}^{(2)}}$, we obtain $\frac{a_{i}}{B_{\alpha, N}^{\prime}} \frac{1}{\left|\sin \left[\frac{1}{2}\left( \pm \frac{\tau v}{M_{N}^{(2)}}\right)\right]\right|^{2 k \alpha}} \leq \frac{a_{i}}{B_{\alpha, N}^{\prime}} \frac{\pi^{2 k \alpha}}{\left|\frac{\tau}{M_{N}^{(1)}}\right|^{2 k \alpha}}$.

Frome the lemma 2.2 , we obtain 
Signal \& Image Processing: An International Journal (SIPIJ) Vol.12, No.5, October 2021

$$
\frac{a_{i}}{B_{\alpha, N}^{\prime}} \frac{1}{\left.\sin \left[\frac{1}{2}\left(+-\frac{\tau v}{M_{N}^{(2)}}\right)\right]\right|^{2 k \alpha}}=O\left(\frac{\left(M_{N}^{(1)}\right)^{2 k \alpha}}{n^{2 k \alpha-1}}\right) .
$$

Therefore, we get

$$
\frac{2^{\frac{p}{\alpha}}}{1-\frac{M_{N}^{(2)}}{M_{N}^{(1)}}} \int_{M_{N}^{(2)}}^{1} W(v)\left|\psi_{N, 2}\left(\tau \lambda \pm \frac{\tau v}{M_{N}^{(1)}}\right)\right|^{\frac{p}{\alpha}} d v=O\left[\frac{1}{n^{(2 k \alpha-1) \frac{p}{\alpha}}}+\left(\frac{\left(M_{N}^{(1)}\right)^{2 k \alpha}}{n^{2 k \alpha-1}}\right)^{\frac{p}{\alpha}}\right]
$$

Choosing $M_{N}^{(1)}$ such that $\frac{\left(M_{N}^{(1)}\right)^{2 k \alpha}}{n^{2 k \alpha-1}}$ converges to 0 . For example $M_{N}^{(1)}=n^{b}$ with $0<b<1-\frac{1}{2 k \alpha}$. Thus, $\lim _{N \rightarrow+\infty} E\left[f_{N}(\lambda)\right]-[\phi(\lambda)]^{\frac{p}{\alpha}}=0$.

Theorem 4.2 Let $-\Omega<\lambda<\Omega$ such that $\phi(\lambda)>0$. Then, $\left.\operatorname{var}\left[f_{N}(\lambda)\right)\right]$ converges to zero. If $M_{N}^{(1)}=n^{c}$ with $\frac{1}{2 k^{2} \alpha^{2}}<c<\frac{1}{2}$, then $\left.\operatorname{var}\left[f_{N}(\lambda)\right)\right]=\mathrm{O}\left(\frac{1}{n^{(1-2 c)}}\right)$.

\section{Proof}

Fisrt suppose that $\tau \lambda \notin\left\{w_{1}, w_{2}, \cdots, w_{q}\right\}$. It is clear that the variance of $f_{N}$ can be written as follows:

$$
\operatorname{var}\left[f_{N}(\lambda)\right]=\int_{R^{2}} W_{N}^{(1)}(\lambda-u) W_{N}^{(1)}\left(\lambda_{1}-u^{\prime}\right) \operatorname{cov}\left[\hat{I}_{N}(u), \hat{I}_{N}\left(u^{\prime}\right)\right] d u d u^{\prime}
$$

Since $W$ is zero for $|\lambda|>1$, for large $N$, we have

$$
\operatorname{var}\left[f_{N}(\lambda)\right]=\int_{-1}^{1} \operatorname{cov}\left[\hat{I}_{N}\left(\lambda-\frac{x_{1}}{M_{N}}\right), \hat{I}_{N}\left(\lambda-\frac{x_{1}^{\prime}}{M_{N}}\right)\right] W\left(x_{1}\right) W\left(x_{1}^{\prime}\right) d x_{1} d x_{1}^{\prime}
$$

Define two subsets of the $[-1,1]^{2}$ by:

$$
\begin{aligned}
& \text { - } L_{1}=\left\{\left(x_{1}, x_{1}^{\prime}\right) \in[-1,1]^{2} ; \quad\left|x_{1}-x_{1}^{\prime}\right|>\sigma_{N}\right\}, \\
& \text { - } L_{2}=\left\{\left(x_{1}, x_{1}^{\prime}\right) \in[-1,1]^{2} ; \quad\left|x_{1}-x_{1}^{\prime}\right| \leq \sigma_{N}\right\},
\end{aligned}
$$


Signal \& Image Processing: An International Journal (SIPIJ) Vol.12, No.5, October 2021

where $\sigma_{N}$ is a nonnegative real, converging to 0 . We split the integral into an integral over the subregion $L_{2}$ and an integral over $L_{1}: \operatorname{var}\left[f_{N}(\lambda)\right]=\int_{L_{2}}+\int_{L_{1}} \stackrel{\Delta}{=} J_{1}+J_{2}$.

Cauchy-Schwartz inequality and theorem 3.1, give

$$
\begin{gathered}
J_{1} \leq C \int_{\left|x_{1}-x_{1}\right| \leq \sigma_{N}} W\left(x_{1}\right) W\left(x_{1}^{\prime}\right) d x_{1} d x_{1}^{\prime} \text {. where } C \text { is constant. Thus, we obtain } \\
J_{1}=O\left(\sigma_{N}\right)
\end{gathered}
$$

It remains to show that $J_{2}$ converges to zero. For simplicity, we define

$$
\lambda_{1}=\lambda-\frac{x_{1}}{M_{N}^{(1)}} ; \quad \lambda_{2}=\lambda-\frac{x_{1}^{\prime}}{M_{N}^{(1)}}, \text { and } C(\lambda)=\operatorname{cov}\left[\hat{I}_{N}\left(\lambda-\frac{x_{1}}{M_{N}^{(1)}}\right), \hat{I}_{N}\left(\lambda-\frac{x_{1}^{\prime}}{M_{N}^{(1)}}\right)\right]
$$

We first show that $C(\lambda)$ converges to zero uniformly in $x_{1}, x_{1}^{\prime} \in[-1,1]$. Indeed, from lemma 2.3, we have

$$
E \hat{I}_{N}(v)-\hat{I}_{N}(v)=F_{p, \alpha}^{-1}\left[C_{\alpha}\right]^{-p / \alpha} \int_{-\infty}^{\infty} \frac{\operatorname{Re}\left(e^{i u I_{N}(v)}\right)-e^{-C_{\alpha}|u|^{\alpha} \psi_{N}(v)}}{|u|^{1+p}} d u
$$

Thus, the expression of the covariance becomes

$$
\begin{aligned}
C(\lambda)= & F_{p, \alpha}^{-2} C_{\alpha}^{-\frac{2 p}{\alpha}} \int_{R^{2}} E\left[\prod_{k=1}^{2} \cos \left(u_{k} I_{N}\left(\lambda_{k}\right)\right)\right] \\
& -\exp \left\{-C_{\alpha} \sum_{k=1}^{2}\left|u_{k}\right|^{\alpha} \psi_{N}\left(\lambda_{k}\right)\right\} \frac{d u_{1} d u_{2}}{\left|u_{1} u_{2}\right|^{1+p}}
\end{aligned}
$$

Using the following equality: $2 \cos x \cos y=\cos (x+y)+\cos (x-y)$, we have

$$
\begin{aligned}
E\left[\prod_{k=1}^{2}\left(\cos u_{k} I_{N}\left(\lambda_{k}\right)\right)\right] & =\frac{1}{2} \exp \left[-C_{\alpha} \int\left|(\tau)^{\frac{1}{\alpha}} \sum_{k=1}^{2} u_{k} H_{N}\left(\tau \lambda_{k}-\tau v\right)\right|^{\alpha} d \mu(v)\right] \\
& +\frac{1}{2} \exp \left[-C_{\alpha} \int\left|(\tau)^{\frac{1}{\alpha}} \sum_{k=1}^{2}(-1)^{k+1} u_{k} H_{N}\left(\tau \lambda_{k}-\tau v\right)\right|^{\alpha} d \mu(v)\right] .
\end{aligned}
$$

By substituting the expression for $C(\lambda)$ and changing the variable $u_{2}$ to $\left(-u_{2}\right)$ in the second term, we obtain

$$
C(\lambda)=F_{p, \alpha}^{-2} C_{\alpha}^{-\frac{2 p}{\alpha}} \int_{R^{2}}\left(e^{-K}-e^{-K^{\prime}}\right) \frac{d u_{1} d u_{2}}{\left|u_{1} u_{2}\right|^{1+p}}
$$


where $K=C_{\alpha} \int_{R}\left|(\tau)^{\frac{1}{\alpha}} \sum_{k=1}^{2} u_{k} H_{N}\left(\tau \lambda_{k}-\tau v\right)\right|^{\alpha} d \xi(v)$ and

$K^{\prime}=C_{\alpha} \tau \sum_{k=1}^{2}\left|u_{k}\right|^{\alpha} \int_{R}\left|H_{N}\left(\tau \lambda_{k}-\tau v\right)\right|^{\alpha} \xi(v) d v$

Since $K, K^{\prime}>0,\left|e^{-K}-e^{-K^{\prime}}\right| \leq\left|K-K^{\prime}\right| \exp \left\{\left|K-K^{\prime}\right|-K^{\prime}\right\}$, we obtain:

$\left|K-K^{\prime}\right| \leq 2 C_{\alpha} \tau|u|^{\frac{\alpha}{2}} Q_{N}\left(\lambda_{1} ; \lambda_{2}\right)$, where

$Q_{N}\left(\lambda_{1} ; \lambda_{2}\right)=\int_{-\Omega}^{\Omega}\left|H_{N}\left(\tau \lambda_{1}-\tau u\right)\right|^{\frac{\alpha}{2}}\left|H_{N}\left(\tau \lambda_{2}-\tau u\right)\right|^{\frac{\alpha}{2}} d \xi(u)$

Now, let us show that $Q_{N}\left(\lambda_{1} ; \lambda_{2}\right)$ converges to zero. Indeed, since $\phi$ is bounded on $[-\Omega, \Omega]$, we have

$$
\begin{aligned}
Q_{N}\left(\lambda_{1} ; \lambda_{2}\right) & \leq \sup (\phi) \int_{-\Omega}^{\Omega}\left|H_{N}\left(\tau \lambda_{1}-\tau u\right) H_{N}\left(\tau \lambda_{2}-\tau u\right)\right|^{\frac{\alpha}{2}} d u \\
& +\sum_{i=1}^{q} c_{i}\left|H_{N}\left(\tau \lambda_{1}-\tau w_{i}\right) H_{N}\left(\tau \lambda_{2}-\tau w_{i}\right)\right|^{\frac{\alpha}{2}}
\end{aligned}
$$

From the definition of $H_{N}$, we can write

$$
\int_{-\Omega}^{\Omega}\left|H_{N}\left(\tau \lambda_{1}-\tau v\right) H_{N}\left(\tau \lambda_{2}-\tau v\right)\right|^{\frac{\alpha}{2}} d v=\left.\int_{-\Omega}^{\Omega} \frac{1}{B_{\alpha, N}^{\prime}}\left|\frac{\sin \left[\frac{n}{2}\left(\tau \lambda_{1}-\tau v\right)\right]}{\sin \left[\frac{1}{2}\left(\tau \lambda_{1}-\tau v\right)\right]}\right|\left|\frac{\sin \left[\frac{n}{2}\left(\tau \lambda_{2}-\tau v\right)\right]}{\sin \left[\frac{1}{2}\left(\tau \lambda_{2}-\tau v\right)\right]}\right|\right|^{k \alpha} d v .
$$

a) First step:

We show that the denominators of the first and second terms under the last integral do not vanish for the same $v$, so we suppose that $v$ exists, belonging to $[-\Omega, \Omega]$ and $z, z^{\prime} \in Z$ such as: $\tau \lambda_{1}-\tau v=2 z \pi$ and $\tau \lambda_{2}-\tau v=2 z^{\prime} \pi$. Since $\lambda_{1} \not \lambda_{2}$, then $z$ and $z^{\prime}$ are different.

Therefore, $\quad z-z^{\prime}=\frac{\tau}{2 \pi}\left(\lambda_{1}-\lambda_{2}\right)$. Hence, $\quad\left|z-z^{\prime}\right|=\frac{1}{w}\left|\lambda_{1}-\lambda_{2}\right|$. As $\quad \lim _{N \rightarrow \infty}\left|\lambda_{1}-\lambda_{2}\right|=0$, consequently, for a large $N$ we get: $|z-z|<\frac{1}{2}$.

Thus, we obtain a contradiction with the fact that $z$ and $z^{\prime}$ are different integers.

b) Second step:

Asuume there exist $q$ points, $V_{1}, V_{2}, \cdots, V_{q} \in[-\Omega, \Omega]$ such as: 
Signal \& Image Processing: An International Journal (SIPIJ) Vol.12, No.5, October 2021 for $j=1,2, \cdots, q \quad \tau \lambda_{1}-\tau V_{j} \in 2 \pi Z$ then $\frac{\lambda_{1}}{w}-\frac{V_{j}}{w} \in Z$, and we assume that there exist $q^{\prime}$ points $V_{1}^{\prime}, V_{2}^{\prime}, \cdots, V_{q^{\prime}}^{\prime} \in[-\Omega, \Omega]$ such that for $i=1,2, \cdots, q^{\prime} \quad \frac{\lambda_{2}}{w}-\frac{V_{i}^{\prime}}{w} \in Z$. Showing that, $\left|V_{j}\right| \neq \Omega$, $\left|V_{i}^{\prime}\right| \neq \Omega$ for $1 \leq j \leq q$ and $1 \leq i \leq q^{\prime}$. Indeed, $-1<\frac{\lambda-\Omega}{w}<0$ and $0<\frac{\lambda+\Omega}{w}<1$ because $w>2 \Omega$. Hence $\frac{\lambda-\Omega}{w} \notin Z$ and $\frac{\lambda+\Omega}{w} \notin Z$. For a large $N$, we get that $\left[\frac{\lambda-\Omega}{w}\right]_{E}<\frac{\lambda_{1}}{w}-\frac{\Omega}{w}<1+\left[\frac{\lambda-\Omega}{w}\right]_{E}, \quad$ where $[x]_{E}$ is the integer part of $x$. Hence, $\frac{\lambda_{1}}{w}-\frac{\Omega}{w} \notin Z$. In the same manner, we show that $\frac{\lambda_{1}}{w}+\frac{\Omega}{w} \notin Z$. Similarly, it can be shown that $\frac{\lambda_{2}+\Omega}{w} \notin Z$. Thus, $\left|V_{j}\right| \neq \Omega$ and $\left|V_{i}^{\prime}\right| \neq \Omega$.

c) Third step:

We classify $V_{j}$ and $V_{i}^{\prime}$ by increasing order: $-\Omega<V_{j_{1}}<V_{j_{2}}<\cdots<V_{j},<\Omega$, and we write the integral in the following manner:

$$
\int_{-\Omega}^{\Omega}\left|\frac{\sin \left[\frac{n}{2}\left(\tau \lambda_{1}-\tau \nu\right)\right]}{\sin \left[\frac{1}{2}\left(\tau \lambda_{1}-\tau v\right)\right]}\right| \frac{\sin \left[\frac{n}{2}\left(\tau \lambda_{2}-\tau v\right)\right]}{\sin \left[\frac{1}{2}\left(\tau \lambda_{2}-\tau_{1} v\right)\right]} \mid d v=I_{1}+\sum_{i=1}^{q+q^{\prime}} I_{2, i}+\sum_{i=1}^{q \alpha} I_{3, i}+I_{4}
$$

where 
Signal \& Image Processing: An International Journal (SIPIJ) Vol.12, No.5, October 2021

$$
\begin{aligned}
& I_{1}=\left.\int_{-\Omega_{1}}^{V_{j_{1}}-\delta(N)}\left|\frac{\sin \left[\frac{n}{2}\left(\tau \lambda_{1}-\tau v\right)\right]}{\sin \left[\frac{1}{2}\left(\tau \lambda_{1}-\tau v\right)\right]}\right| \frac{\sin \left[\frac{n}{2}\left(\tau \lambda_{2}-\tau v\right)\right]}{\sin \left[\frac{1}{2}\left(\tau \lambda_{2}-\tau v\right)\right]}\right|^{k \alpha} d v \\
& I_{2, i}=\left.\int_{V_{j_{i}}-\delta(N)}^{V_{j_{i}}+\delta(N)}\left|\frac{\sin \left[\frac{n}{2}\left(\tau \lambda_{1}-\tau v\right)\right]}{\sin \left[\frac{1}{2}\left(\tau \lambda_{1}-\tau v\right)\right]}\right| \frac{\sin \left[\frac{n}{2}\left(\tau \lambda_{2}-\tau v\right)\right]}{\sin \left[\frac{1}{2}\left(\tau \lambda_{2}-\tau v\right)\right]}\right|^{k \alpha} d v \\
& I_{3, i}=\int_{V_{j_{i}}+\delta(N)}^{V_{j_{i+1}}-\delta(N)}\left|\frac{\sin \left[\frac{n}{2}\left(\tau \lambda_{1}-\tau v\right)\right]}{\sin \left[\frac{1}{2}\left(\tau \lambda_{1}-\tau v\right)\right]}\right| \frac{\sin \left[\frac{n}{2}\left(\tau \lambda_{2}-\tau v\right)\right]}{\sin \left[\frac{1}{2}\left(\tau \lambda_{2}-\tau v\right)\right]}||^{k \alpha} d v \\
& I_{4}=\int_{V_{j}, q^{\prime}}^{\Omega}\left|\frac{\sin \left[\frac{n}{2}\left(\tau \lambda_{1}-\tau v\right)\right]}{\sin \left[\frac{1}{2}\left(\tau \lambda_{1}-\tau v\right)\right]}\right| \frac{\sin \left[\frac{n}{2}\left(\tau \lambda_{2}-\tau v\right)\right]}{\sin \left[\frac{1}{2}\left(\tau_{1} \lambda_{2}-\tau v\right)\right]}||^{k \alpha} d v
\end{aligned}
$$

where $\delta(N)$ is a nonnegative real number converging to zero and satisfying:

$$
\begin{aligned}
& -\Omega<V_{j_{1}}-\delta(N)<V_{j_{1}}+\delta(N)<V_{j_{2}}-\delta(N)<V_{j_{2}}+\delta(N)<\ldots \ldots<V_{j_{q+q^{\prime}}}-\delta(N)<V_{j},+\delta(N)<\Omega, \mathrm{a} \\
& \text { nd } \delta(N)<\left|\frac{\lambda_{1}-\lambda_{2}}{2}\right| .
\end{aligned}
$$

Showing that the first integral converges to zero. For a large $N$, it easy to see that $\lambda_{1}<\Omega$. Without loss of generality, we assume that $\tau \lambda_{1}-\tau V_{j_{i}}=2 k_{i} \pi$ for all $i$, with $k_{i} \in Z$. The fact that there is no $v$ between $-\Omega$ and $V_{j_{1}}-\delta(N)$ on which the denominators are vanishing, gives

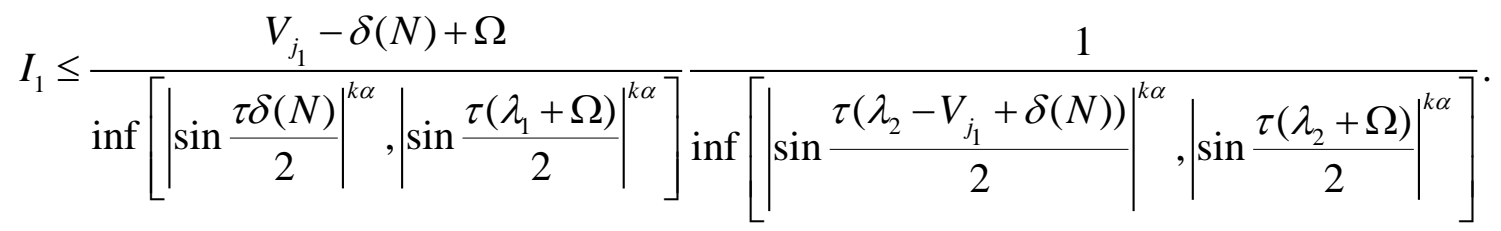

Substituting $V_{j_{1}}$ in the last inequality, we get

$$
\left|\sin \frac{\tau\left(\lambda_{2}-V_{j_{1}}+\delta(N)\right)}{2}\right|^{k \alpha}=\left|\sin \frac{\tau\left|\lambda_{2}-\lambda_{1}+\delta(N)\right|}{2}\right|^{k \alpha} \text {. For a large } N \text {, we obtain }
$$


Signal \& Image Processing: An International Journal (SIPIJ) Vol.12, No.5, October 2021

$$
\frac{\tau\left|\lambda_{2}-\lambda_{1}+\delta(N)\right|}{2} \leq \tau \Omega+\frac{\tau \delta(N)}{2}<\pi-\frac{\tau \delta(N)}{2} .
$$

On the other hand, two cases are possible:

1. $\lambda_{2}-\lambda_{1}>0$, then we have $\left|\lambda_{2}-\lambda_{1}+\delta(N)\right|=\lambda_{2}-\lambda_{1}+\delta(N)>\delta(N)$

2. $\lambda_{2}-\lambda_{1}<0$, since $\left|\lambda_{2}-\lambda_{1}\right|>2 \delta(N)$, we have

$$
\left|\lambda_{2}-\lambda_{1}+\delta(N)\right|=\lambda_{1}-\lambda_{2}-\delta(N)>\delta(N) \text {. }
$$

Therefore, $\frac{\tau \delta(N)}{2}<\frac{\tau\left|\lambda_{2}-\lambda_{1}+\delta(N)\right|}{2}<\pi-\frac{\tau \delta(N)}{2}$. For a large $N$, we have $\tau \delta(N)<2 \pi-\tau\left(\lambda_{1}+\Omega\right)$ and $\tau \delta(N)<2 \pi-\tau\left(\lambda_{2}+\Omega\right)$. Then,

$$
\frac{\tau \delta(N)}{2}<\frac{\tau\left(\lambda_{1}+\Omega\right)}{2}<\pi-\frac{\tau \delta(N)}{2} \quad \text { and } \quad \frac{\tau \delta(N)}{2}<\frac{\tau\left(\lambda_{2}+\Omega\right)}{2}<\pi-\frac{\tau \delta(N)}{2} .
$$

Therefore,

$$
I_{1} \leq \frac{V_{j_{1}}-\delta(N)+\Omega}{\left|\sin \frac{\tau \delta(N)}{2}\right|^{2 k \alpha}}
$$

For the integral $I_{2, i}$, we bound the first fraction under integral by $n^{k \alpha}$ :

$$
I_{2, i} \leq n^{k \alpha} \int_{V_{j_{i}}-\delta(N)}^{V_{j_{i}}+\delta(N)} \frac{1}{\left|\sin \left[\frac{1}{2}\left(\tau \lambda_{2}-\tau v\right)\right]\right|^{k \alpha}} d v \text {. By substituting for } V_{j_{i}} \text { in the last }
$$

inequality and putting $v=u-\frac{2 k \pi}{\tau}$, we get

$$
\begin{gathered}
I_{2, i} \leq n^{k \alpha} \int_{\lambda_{1}-\delta(N)}^{\lambda_{1}+\delta(N)} \frac{1}{\left|\sin \left[\frac{1}{2}\left(\tau \lambda_{2}-\tau u\right)\right]\right|} d u \text {. Since }\left|\lambda_{1}-u\right|<\delta(N), \text { it is easy to see that } \\
\left|\lambda_{2}-u\right| \geq\left|\lambda_{2}-\lambda_{1}\right|-\left|\lambda_{1}-u\right| \geq\left|\lambda_{2}-\lambda_{1}\right|-\delta(N)>\frac{\left|\lambda_{2}-\lambda_{1}\right|}{2}
\end{gathered}
$$

Since $\delta(N)$ converges to zero, for a large $N$ we have $\delta(N)<\frac{2}{\tau}\left(\pi-\frac{\tau}{2}\left|\lambda_{2}-\lambda_{1}\right|\right)$, therefore $0<\tau \frac{\left|\lambda_{2}-\lambda_{1}\right|}{4}<\tau \frac{\left|\lambda_{2}-u\right|}{2}<\tau \frac{\left|\lambda_{2}-\lambda_{1}\right|+\delta(N)}{2}<\pi$. Consequently: 
Signal \& Image Processing: An International Journal (SIPIJ) Vol.12, No.5, October 2021

$$
I_{2, i} \leq \frac{2 \delta(N) n^{k \alpha}}{\inf \left[\sin \frac{\left|\lambda_{1}-\lambda_{2}\right| \tau}{4} ; \sin \frac{\left|\lambda_{1}-\lambda_{2}\right| \tau+\delta(N) \tau}{2}\right]}
$$

where $\left|\lambda_{2}-\lambda_{1}\right|=\left|\frac{x_{2}-x_{1}}{M_{N}}\right|$. Then, for a large $N$, we have $\frac{3 \tau}{2}\left|\lambda_{2}-\lambda_{1}\right|<2 \pi$. Therefore, $\tau \frac{\left|\lambda_{2}-\lambda_{1}\right|}{4}<\frac{\tau\left|\lambda_{2}-\lambda_{1}\right|+\tau \delta(N)}{2}<\pi-\tau \frac{\left|\lambda_{2}-\lambda_{1}\right|}{4}$. Thus, we bound the integral as follows:

$$
I_{2, i} \leq \frac{2 \delta(N) n^{k \alpha}}{\left|\sin \frac{\left.\tau\left|\lambda_{2}-\lambda_{1}\right|\right)}{4}\right|^{k \alpha}}
$$

Since there is no $v$ between $V_{j_{i}}+\delta(N)$ and $V_{j_{i+1}}-\delta(N)$ on which the denominators are vanishing, we get:

$$
I_{3, i} \leq \frac{V_{j_{i+1}}-V_{j_{i}}-2 \delta(N)}{A \times B}
$$

where

$$
\begin{aligned}
& A=\inf \left(\left|\sin \frac{\tau \lambda_{1}-\tau V_{j_{i}}-\tau \delta(N)}{2}\right|^{k \alpha},\left|\sin \frac{\tau \lambda_{1}-\tau V_{j_{i+1}}+\tau \delta(N)}{2}\right|^{k \alpha}\right) \\
& B=\inf \left(\left|\sin \frac{\tau \lambda_{2}-\tau V_{j_{i}}-\tau \delta(N)}{2}\right|^{k \alpha},\left|\sin \frac{\tau \lambda_{2}-\tau V_{j_{i+1}}+\tau \delta(N)}{2}\right|^{k \alpha}\right)
\end{aligned}
$$

It follows from the hypothesis on $\delta(N)$ that

$$
\frac{\tau \delta(N)}{2}<\frac{\tau\left|\lambda_{1}-\lambda_{2}\right|}{2}-\frac{\tau|\delta(N)|}{2}<\frac{\tau\left|\lambda_{1}-\lambda_{2}-\delta(N)\right|}{2}<\frac{\tau\left|\lambda_{1}-\lambda_{2}\right|}{2}+\frac{\tau|\delta(N)|}{2}<\pi-\frac{\tau \delta(N)}{2} .
$$

The definition of $V_{j_{i}}$ gives 
Signal \& Image Processing: An International Journal (SIPIJ) Vol.12, No.5, October 2021

$$
\begin{aligned}
& \quad \frac{1}{\left|\sin \frac{\tau \lambda_{2}-\tau V_{j_{i}}-\tau \delta(N)}{2}\right|^{k \alpha}}<\frac{1}{\left|\sin \frac{\tau \delta(N)}{2}\right|^{k \alpha}} \text {. Similarly we bound the other terms: } \\
& I_{3, i} \leq \frac{V_{j_{i+1}}-V_{j_{i}}-2 \delta(N)}{\left|\sin \frac{\tau \delta(N)}{2}\right|^{2 k \alpha}} .
\end{aligned}
$$

We can show by same majoration that :

$$
\begin{aligned}
& I_{4} \leq \frac{V_{j_{i+1}}-V_{j_{i}}-2 \delta(N)}{E \times F} \text {, where } \\
& E=\inf \left(\left|\sin \frac{\tau \lambda_{1}-\tau \Omega}{2}\right|^{k \alpha},\left|\sin \frac{\tau \lambda_{1}-\tau V_{j_{q+q^{\prime}}}-\tau \delta(N)}{2}\right|^{k \alpha}\right) \\
& F=\inf \left(\left|\sin \frac{\tau \lambda_{2}-\tau \Omega}{2}\right|^{k \alpha},\left|\sin \frac{\tau \lambda_{2}-\tau V_{j_{q+q^{\prime}}}-\tau \delta(N)}{2}\right|^{k \alpha}\right)
\end{aligned}
$$

Since $\delta(N)$ converges to zero, for a large $N$, we have $\delta(N)<\frac{2}{\tau}\left(\pi-\frac{\tau}{2}\left|\lambda_{1}-\Omega\right|\right)$, and $\delta(N)<\frac{2}{\tau}\left(\pi-\frac{\tau}{2}\left|\lambda_{1}-\lambda_{2}\right|\right)$. It follows that: $I_{4} \leq \frac{\Omega-V_{j_{q+q^{\prime}}}-\delta(N)}{\left|\sin \frac{\tau \delta(N)}{2}\right|^{2 k \alpha}}$. We recapitulate, from the previous increases, we obtain

$$
\begin{aligned}
& \int_{-\Omega}^{\Omega}\left|\frac{\sin \left[\frac{n}{2}\left(\tau \lambda_{1}-\tau v\right)\right]}{\sin \left[\frac{1}{2}\left(\tau \lambda_{1}-\tau v\right)\right]}\right| \frac{\sin \left[\frac{n}{2}\left(\tau \lambda_{2}-\tau \nu\right)\right]}{\sin \left[\frac{1}{2}\left(\tau \lambda_{2}-\tau v\right)\right]} \mid d v \leq \frac{\Omega+V_{j_{1}}-\delta(N)}{\left|\sin \frac{\tau \delta(N)}{2}\right|^{2 k \alpha}+\sum_{i=1}^{q+q^{\prime}} \frac{2 n^{k \alpha} \delta(N)}{\left|\sin \frac{\tau\left|\lambda_{2}-\lambda_{1}\right|}{4}\right|^{k \alpha}}} \\
&+\sum_{i=1}^{q+q^{\prime}-1} \frac{V_{j_{i+1}}-V_{j_{i}}-\delta(N)}{\left|\sin \frac{\tau \delta(N)}{2}\right|^{2 k \alpha}}+\frac{\Omega-V_{j_{q+q^{\prime}}}-\delta(N)}{\left|\sin \frac{\tau \delta(N)}{2}\right|^{2 k \alpha}}
\end{aligned}
$$

After simplification, we have 
Signal \& Image Processing: An International Journal (SIPIJ) Vol.12, No.5, October 2021

$\int_{-\Omega}^{\Omega}\left|\frac{\sin \left[\frac{n}{2}\left(\tau \lambda_{1}-\tau \nu\right)\right]}{\sin \left[\frac{1}{2}\left(\tau \lambda_{1}-\tau \nu\right)\right]}\right|\left|\frac{\sin \left[\frac{n}{2}\left(\tau \lambda_{2}-\tau \nu\right)\right]}{\sin \left[\frac{1}{2}\left(\tau \lambda_{2}-\tau \nu\right)\right]}\right| d v \leq \frac{2 \Omega-2\left(q+q^{\prime}+1\right) \delta(N)}{\left|\sin \frac{\tau \delta(N)}{2}\right|^{2 k \alpha}}+\frac{2 n^{k \alpha} \delta(N)\left(q+q^{\prime}\right)}{\left|\sin \frac{\tau\left|\lambda_{2}-\lambda_{1}\right|}{4}\right|^{k \alpha} .}$

Using the following inequality $\left|\sin \frac{x}{2}\right| \geq \frac{|x|}{\pi}$, we get

$\left.\int_{-\Omega}^{\Omega}\left|\frac{\sin \left[\frac{n}{2}\left(\tau \lambda_{1}-\tau \nu\right)\right]}{\sin \left[\frac{1}{2}\left(\tau \lambda_{1}-\tau \nu\right)\right]}\right|\left|\frac{\sin \left[\frac{n}{2}\left(\tau \lambda_{2}-\tau \nu\right)\right]}{\sin \left[\frac{1}{2}\left(\tau \lambda_{2}-\tau \nu\right)\right]}\right|\right|^{k \alpha} d \nu \leq \frac{2 \Omega \pi^{2 k \alpha}}{(\tau \delta(N))^{2 k \alpha}}+\frac{2 n^{k \alpha} \delta(N)\left(q+q^{\prime}\right)(2 \pi)^{k \alpha}}{\left(\frac{\tau\left|x_{2}-x_{1}\right|}{M_{N}}\right)^{k \alpha}}$.

The lemma 2.1 gives

$$
\begin{array}{r}
\frac{1}{B_{\alpha, N}^{\prime}} \int_{-\Omega}^{\Omega}\left|\frac{\sin \left[\frac{n}{2}\left(\tau \lambda_{1}-\tau \nu\right)\right]}{\sin \left[\frac{1}{2}\left(\tau \lambda_{1}-\tau \nu\right)\right]}\right|\left|\frac{\sin \left[\frac{n}{2}\left(\tau \lambda_{2}-\tau \nu\right)\right]}{\sin \left[\frac{1}{2}\left(\tau \lambda_{2}-\tau \nu\right)\right]}\right| d \nu \leq \\
\frac{1}{\pi}\left(\frac{\pi}{2}\right)^{2 k \alpha}\left(\frac{2 \Omega \pi^{2 k \alpha}}{n^{2 k \alpha-1}(\tau \delta(N))^{2 k \alpha}}+\frac{2 \delta(N)\left(q+q^{\prime}\right)(2 \pi)^{k \alpha}}{n^{k \alpha-1}\left(\frac{\tau \sigma_{N}}{M_{N}}\right)^{k \alpha}}\right) .
\end{array}
$$

In order to obtain the convergence of the last expression to zero, we choose $\delta(N)=n^{-\beta}, \quad \beta>0$, such as

$$
\lim _{n \rightarrow \infty} \frac{n^{2 k \alpha \beta}}{n^{2 k \alpha-1}}=0 \quad \text { and } \quad \lim _{n \rightarrow \infty} \frac{1}{n^{\beta+k \alpha-1}\left(\frac{\sigma_{N}}{M_{N}}\right)^{k \alpha}}=0
$$

Thus, from (18) $Q_{N}$ converges to zero. On the other hand,

$$
C(\lambda) \leq F_{p, \alpha}^{-2} C_{\alpha}^{-\frac{2 p}{\alpha}} \int_{-\infty}^{\infty} \int_{-\infty}^{\infty}\left|K-K^{\prime}\right| e^{\left|K-K^{\prime}\right|-K^{\prime}} \frac{d u_{1} d u_{2}}{\left|u_{1} u_{2}\right|^{1+p}},
$$

where $\left|K-K^{\prime}\right|-K^{\prime} \leq-C_{\alpha} \sum_{k^{\prime}=1}^{2}\left|u_{k^{\prime}}\right|^{\alpha}\left|\psi_{N}\left(\lambda_{k^{\prime}}\right)-\tau Q_{N}\left(\lambda_{1} ; \lambda_{2}\right)\right|$.

We denote by: $\delta_{\left(N, k^{\prime}\right)}=\psi_{N}\left(\lambda_{k^{\prime}}\right)-\tau Q_{N}\left(\lambda_{1} ; \lambda_{2}\right)$. It follows from (18) and (20) that $\delta_{\left(N, k^{\prime}\right)}$ converges to $\phi(\lambda)$. Hence, 


$$
C(\lambda) \leq F_{p, a}^{-2} C_{\alpha}^{-\frac{2 p}{\alpha}} 2 \tau C_{\alpha} Q_{N}\left(\lambda_{1} ; \lambda_{2}\right) 4 \prod_{k^{\prime}=1}^{2} \int_{0}^{\infty} \exp \left[-C_{\alpha}\left(u_{k^{\prime}}\right)^{\alpha}\left|\delta_{\left(N, k^{\prime}\right)}\right|\right] \frac{d u_{k^{\prime}}}{\left(u_{k^{\prime}}\right)^{1+p-\frac{\alpha}{2}}} .
$$

Putting $\quad u_{k^{\prime}}\left(\delta_{N, k^{\prime}}\right)^{\frac{1}{\alpha}}=v$, we obtain

$$
C(\lambda) \leq F_{p, \alpha}^{-2} C_{\alpha}^{-\frac{2 p}{\alpha}} 2 \tau C_{\alpha} \frac{Q_{N}\left(\lambda_{1} ; \lambda_{2}\right)}{\left[\delta_{(N, 1)} \delta_{(N, 2)}\right]^{\frac{1}{2}-\frac{p}{\alpha}}}\left(\int_{-\infty}^{\infty} \frac{e^{-C_{\alpha}|\nu|^{\alpha}}}{|v|^{1+p-\frac{\alpha}{2}}} d v\right)^{2}
$$

Since $\phi(\lambda)>0, C(\lambda)$ converges uniformly in $x_{1}, x_{1}^{\prime} \in[-1,1]$ to zero. From (20), we obtain $J_{2}=O\left(\frac{1}{n^{2 k \alpha(1-\beta)-1}}+\frac{1}{n^{\beta+k \alpha-1}\left(\frac{\sigma_{N}}{M_{N}}\right)^{k \alpha}}\right)$. Thus, $\operatorname{var}\left[f_{N}(\lambda)\right]$ converges to zero and then, $f_{N}(\lambda)$ is an asymptotically unbiased and consistent estimator.

\section{Conclusions}

This paper gives an estimate of the spectral density of a mixed continuous-time stable process from observations at discrete instants. To avoid the phenomenon of aliasing we have assumed that the spectral density is a compact support. The applications of these processes are found in various fields. For example:

- The study of soil cracking where the observed signal is the resistance of the soil. This signal encounters random jumps due to the encounter of certain stones in the ground. The spectral measurement will therefore be composed of two parts, one continuous and the other discrete. This last corresponds to the resistance jumps encountered during the measurement.

- The growth of fruits on a tree can be seen as a continuous distribution, and when there is a fall of a fruit, the other fruits remaining on the tree absorb more energy and their growth will have a jump in value.

As perspective of this work is to find the optimum smoothing parameters to have a better rate of convergence. For this purpose, the cross-validation method will be the most appropriate tool.

We intend to complete this work by studying the case where the process is observed with random errors. For this, we will use the deconvolution methods known for their efficiency in the presence of random errors.

We think to give an estimator of the mode of the spectral density representing the frequency where the spectral density reaches the maximum of energy. 
Signal \& Image Processing: An International Journal (SIPIJ) Vol.12, No.5, October 2021

\section{REFERENCES}

[1] S. Cambanis, (1983)“Complex symetric stable variables and processes” In P.K.SEN, ed, Contributions to Statistics": Essays in Honour of Norman L. Johnson North-Holland. New York,(P. K. Sen. ed.), pp. 63-79

[2] S. Cambanis, and M. Maejima (1989). "Two classes of self-similar stable processes with stationary increments". Stochastic Process. Appl. Vol. 32, pp. 305-329

[3] M.B. Marcus and K. Shen (1989). "Bounds for the expected number of level crossings of certain harmonizable infinitely divisible processes". Stochastic Process. Appl., Vol. 76, no. 1 pp 1-32.

[4] E. Masry, S. Cambanis (1984). "Spectral density estimation for stationary stable processes". Stochastic processes and their applications, Vol. 18, pp. 1-31 North-Holland.

[5] G. Samorodnitsky and M. Taqqu (1994). "Stable non Gaussian processes ». Chapman and Hall, New York.

[6] K., Panki and S. Renming (2014). "Stable process with singular drift”. Stochastic Process. Appl., Vol. 124, no. 7, pp. 2479-2516

[7] C. Zhen-Qing and W. Longmin (2016). "Uniqueness of stable processes with drift." Proc. Amer. Math. Soc., Vol. 144, pp. 2661-2675

[8] K. Panki, K. Takumagai and W. Jiang (2017). "Laws of the iterated logarithm for symmetric jump processes". Bernoulli, Vol. 23, n 4 pp. 2330-2379.

[9] M. Schilder (1970). "Some Structure Theorems for the Symmetric Stable Laws". Ann. Math. Statist., Vol. 41, no. 2, pp. 412-421.

[10] R. Sabre (2012b). "Missing Observations and Evolutionary Spectrum for Random”. International Journal of Future Generation Communication and Networking, Vol. 5, n 4, pp. 55-64.

[11] E. Sousa (1992). "Performance of a spread spectrum packet radio network link in a Poisson of interferences". IEEE Trans. Inform. Theory, Vol. 38, pp. 1743-1754

[12] M. Shao and C.L. Nikias (1993). "Signal processing with fractional lower order moments: Stable processes and their applications", Proc. IEEE, Vol.81, pp. 986-1010

[13] C.L. Nikias and M. Shao (1995). "Signal Processing with Alpha-Stable Distributions and Applications". Wiley, New York

[14] S. Kogon and D. Manolakis (1996). "Signal modeling with self-similar alpha- stable processes: The fractional levy motion model”. IEEE Trans. Signal Processing, Vol 44, pp. 1006-1010

[15] N. Azzaoui, L. Clavier, R. Sabre, (2002). "Path delay model based on stable distribution for the 60GHz indoor channel” IEEE GLOBECOM, IEEE, pp. 441-467

[16] J.P. Montillet and Yu. Kegen (2015). "Modeling Geodetic Processes with Levy alpha-Stable Distribution and FARIMA", Mathematical Geosciences. Vol. 47, no. 6, pp. 627-646.

[17] M. Pereyra and H. Batalia (2012). "Modeling Ultrasound Echoes in Skin Tissues Using Symmetric alpha-Stable Processes". IEEE Transactions on Ultrasonics, Ferroelectrics, and Frequency Control, Vol. 59, $\mathrm{n}^{\circ}$. 1, pp. 60-72.

[18] X. Zhong and A.B. Premkumar (2012). "Particle filtering for acoustic source tracking in impulsive noise with alpha-stable process". IEEE Sensors Journal, Vol. 13, no. 2, pp. 589 - 600.

[19] Wu. Ligang and W. Zidong (2015). "Filtering and Control for Classes of Two-Dimensional Systems". The series Studies in Systems of, Decision and Control, Vol.18, pp. 1-29.

[20] N. Demesh (1988). "Application of the polynomial kernels to the estimation of the spectra of discrete stable stationary processes”. (Russian) Akad.Nauk.ukrain. S.S.R. Inst.Mat. Preprint 64, pp. 12-36

[21] F. Brice, F. Pene, and M. Wendler, (2017) "Stable limit theorem for U-statistic processes indexed by a random walk", Electron. Commun. Prob., Vol. 22, no. 9, pp.12-20.

[22] R. Sabre (2019). “Alpha Stable Filter and Distance for Multifocus Image Fusion”. IJSPS, Vol. 7, no. 2, pp. 66-72.

[23] JN. Chen, J.C. Coquille, J.P. Douzals, R. Sabre (1997). "Frequency composition of traction and tillage forces on a mole plough". Soil and Tillage Research, Vol. 44, pp. 67-76.

[24] R. Sabre (1995). "Spectral density estimate for stationary symmetric stable random field", Applicationes Mathematicaes, Vol. 23, n. 2, pp. 107-133

[25] R. Sabre (2012a). "Spectral density estimate for alpha-stable p-adic processes". Revisita Statistica, Vol. 72, n. 4, pp. 432-448.

[26] R. Sabre (2017). "Estimation of additive error in mixed spectra for stable prossess". Revisita Statistica, Vol. LXXVII, $n^{\circ}$. 2, pp. 75-90. 
Signal \& Image Processing: An International Journal (SIPIJ) Vol.12, No.5, October 2021

[27] E. Masry, (1978). "Alias-free sampling: An alternative conceptualization and its applications", IEEE Trans. Information theory, Vol. 24, pp.317-324.

\section{AUTHORS}

Rachid Sabre received the PhD degree in statistics from the University of Rouen, France, in 1993 and Habilitation to direct research (HdR) from the University of Burgundy, Dijon, France, in 2003. He joined Agrosup Dijon, France, in 1995, where he is an Associate Professor. From 1998 through 2010, he served as a member of Institut de Mathématiques de Bourgogne, France. He was a member of the Scientific Council AgroSup Dijon from 2009 to 2013. From 2012 to 2019, he has been a member of Laboratoire Electronic, Informatique, and Image (LE2I), France. Since 2019 he has been a member of Laboratory Biogeosciences UMR CNRS University Burgundy. He is author/co-author of numerous papers in scientific and technical journals and conference proceedings. His research interests lie in areas of statistical process and spectral analysis for signal and image processing. 